\title{
A RADIO DIRECTION FINDER FOR USE ON AIRCRAFT
}

\author{
By Wilbur S. Hinman, Jr.
}

\section{ABSTRACT}

A new type of radio direction finder is described which does away with phasing difficulties by using a single loop antenna having a field pattern modified by dissymmetry in the loop-antenna circuit. Visual indication of the "course" is given, and the direction finder is bilateral and unidirectional. The indication of the "course" is not distorted at any volume level, including complete overload. The characteristics of the incoming signals are not destroyed when "on-course" indications are received, but a loud audio note is produced when the loop antenna is turned either side of "course." The direction finder may be added as a unit to any radio receiving set. It operates on any received signal, with modulated or unmodulated waves. The sharpness of indication is readily controllable. This direction finder is based on the idea of reversing a dissymmetrical loop antenna with respect to ground, thereby producing two equal but opposite distorted field patterns. This is accomplished by alternately grounding the ends of a loop antenna through rectifier tubes and applying the voltage induced in the loop antenna by a radio wave to a radio receiver. The output of the radio receiver is also applied to these rectifier tubes, and a zero-center course indicator is so connected that when the loop antenna is grounded at one end, the course indicator deflects right in proportion to the voltage induced in the loop antenna for that condition. When the loop antenna is grounded at the other end, the course indicator deflects left in a similar manner. Thus the course indicator shows directly a comparison of the two field patterns, and gives zero-center course indication.

Flight tests were made using broadcasting stations of Washington, D.C., and Baltimore, Md. Steady and accurate bilateral indication of "course" was given, as well as positive localizing action (by the reversal of the course indicator action). The early models contributing to the final development are briefly discussed and their circuit arrangements are given where of interest.

\section{CONTENTS}

I. Introduction

II. Preliminary models

III. Single loop antenna radio direction finder

1. Principle of operation.

2. The circuit arrangement

3. Construction

4. Tests.

IV. Acknowledgment.

\section{INTRODUCTION}

The need for an accurate marking of the principal air routes of the country has been well met by the installation of radio range beacons. Their operation over a number of years shows quite definitely that they are satisfactorily devised for guiding aircraft. The limitation, however, is that they may only be used on the particular air routes for which they were installed. The itinerant pilot cannot always use them. Thus there is the need for a direction finder operating as 
a radiocompass with which the pilot may be guided to a point to which radio range beacons are not available.

The following general requirements were considered essential for a satisfactory aircraft direction finder:

(1) It must give accurate bilateral indication with no mechanically moving parts. (Moving parts are cumbersome and liable to failure.)

(2) It must be capable of being added as a unit to a standard radio receiver with no adjustments other than tuning and control of volume. (Phasing of currents must be accomplished automatically.)

(3) It must give definite directive sense with no false courses. (That is, there should be one true course, with neither $90^{\circ}$ nor $180^{\circ}$ ambiguity.)

(4) It should operate both on modulated and unmodulated radio waves and should not destroy the characteristics of the received signal.

\section{PRELIMINARY MODELS}

The well-known Robinson direction finder with two crossed-loop antennas was used as a basis for development. The first model consisted of the mechanical reversal of one loop antenna with respect to the other by means of a rotating coil in a coupling system. The output of the radio receiver was applied to a zero-center course-indicating meter through a reversing switch operated in synchronism with the rotating coil. Indication right and left of "course" was obtained, but the $90^{\circ}$ courses introduced by the two crossed-loop antennas and the mechanically moving parts made this model far from promising as a direction finder.

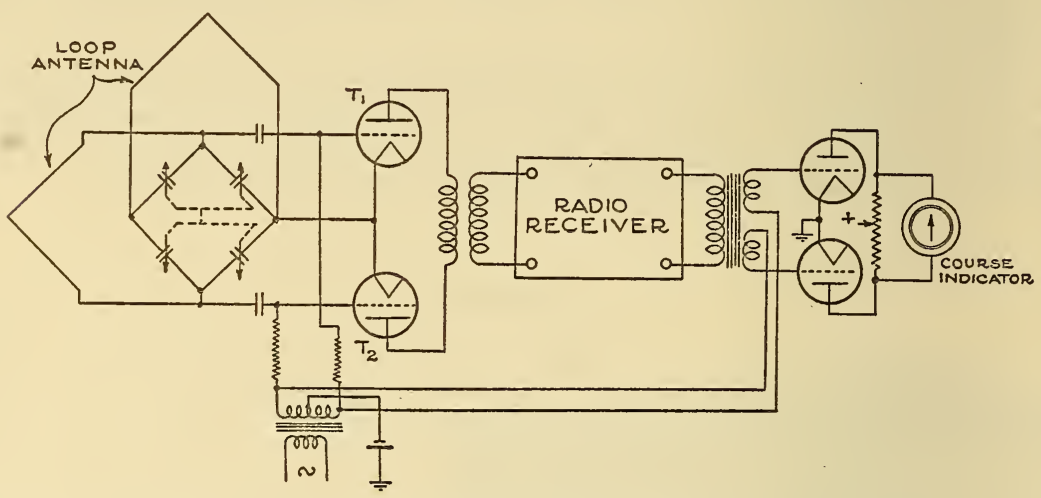

FigURE 1.-Double-loop-antenna direction finder with Bellini-Tosi bridge condenser tuning arrangement and electrical swilching.

Electrical switching was then tested, using the 2 crossed-loop antennas with 2 input and 2 output tubes which were alternately operated and cut off in pairs by an alternating voltage applied to their grids. The loop antennas were tuned by a special bridge condenser arrangement developed by Bellini-Tosi, which seemed especially adaptable to this direction finder by reason of the combination of the currents in the condensers. The circuit arrangement is shown in figure 1. This direction finder also gave indication right and left of "course", but it had $90^{\circ}$ courses. It had the further disadvantage that extreme 
care was necessary in the adjustment and matching of the input and output tubes.

A model was then constructed, using the idea of alternately connecting and disconnecting one loop antenna, and utilizing a special output switching arrangement. The operation is best explained by reference to the circuit arrangement shown in figure 2. The loop

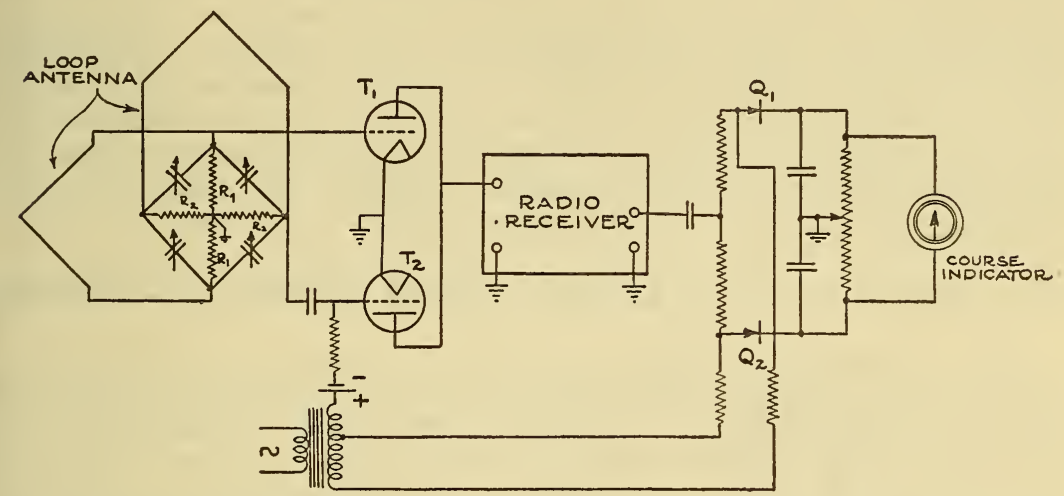

Figure 2.-Double-loop-antenna direction finder with modified Bellini-Tosi bridge condenser tuning arrangement.

antennas and bridge condenser arrangement is similar to that previously described. The currents of the two loop antennas are separated by the four resistors $R_{1} R_{1}, R_{2} R_{2}$. $R_{1} R_{1}$ carry the current of one loop antenna and are applied to $T_{1}$, and $R_{2} R_{2}$ carry the current of the other loop antenna and are applied to $T_{2}$. Tube $T_{2}$ is alternately operated and cut off by means of an alternating voltage applied to its grid, so that the voltage applied to the radio receiver is alternately the voltage of one loop antenna, and the sum or difference of the voltages of both loop antennas. The output device consists of two copper-oxide rectifiers $Q_{1}$ and $Q_{2}$ connected to a zero-center course indicator so that they pass current through the course indicator in opposite directions. Then, if equal alternating voltages opposite in phase are applied to the rectifiers, equal and opposite currents will flow through the course indicator. This alternating voltage is supplied from the source which operates $T_{2}$, so that $T_{2}$ operates with one rectifier and is cut off with the other rectifier. The output voltage of the radio receiver is superimposed on the alternating voltages applied to the rectifiers. Then when a signal is received $Q_{1}$ passes current due to one loop antenna (the other loop antenna being cut off by $\left.T_{2}\right)$, and $Q_{2}$ passes current due to both loop antennas $\left(T_{2}\right.$ being in an operating condition). If the loop antenna whose voltage is applied to $T_{2}$ is at minimum with respect to an incoming radio wave, the loop antenna applied to $T_{1}$ gives the whole signal applied to $Q_{1}$ and $Q_{2}$, and the course indicator is at zero. If, however, the loop antenna supplying $T_{2}$ is not at minimum, then $Q_{1}$ passes current due to the voltage developed in one loop antenna, and $Q_{2}$ passes current due to the voltage developed in both loop antennas. The voltage developed in both loop antennas is either greater or less than that due to one loop antenna, depending on the phase relations of the currents. This gives right and left indications of "course", since for 
one side of "course" the current due to the voltages of the loop antennas will aid and for the other side of "course" they will oppose each other.

While this direction finder gave more promise than any of the other models, the two crossed-loop antennas were cumbersome and the tuning system was bulky. Two false courses are found where the voltage developed in one loop antenna equals the difference between the voltages in both loop antennas, and the position of this course varies with the relative intensity of the two currents. These false courses could, of course, be avoided by the use of an aperiodic vertical antenna in place of one loop antenna. The chief advantage of this direction finder is the automatic phasing of the currents of the two loop antennas, and the simultaneous tuning of both loop antennas. Variations in the input tubes cannot produce course errors.

\section{SINGLE LOOP ANTENNA RADIO DIRECTION FINDER}

The next model of a direction finder was based on the idea of reversing a loop antenna which is dissymmetrical with respect to ground. This was accomplished by alternately grounding the ends of a loop antenna through rectifier tubes and applying the voltage induced in the loop antenna by a radio wave to a radio receiver. A suitable output system synchronized with the input tubes, and applied to a zero-center course indicator was connected to the output of the radio receiver. The course indicator was made to deflect to the right when the loop antenna was grounded at one end, and to the left when the loop antenna was grounded at the other end in proportion to the signal developed for the two conditions. It was immediately evident that this direction finder was worth further development.

\section{PRINCIPLE OF OPERATION}

For a number of years it has been common practice to introduce into a balanced loop antenna sufficient voltage from a vertical antenna to change the normal figure-of-eight field pattern of the loop antenna into a cardioid. Methods for accomplishing this are well known, the requirements being that the vertical antenna current be $90^{\circ}$ out of phase with the loop antenna currents, or in phase with their resultant. Since a loop antenna which is not symmetrical with respect to ground tends to act partially as a vertical antenna, this cardioid type of field pattern can be approached without the use of a vertical antenna. Careful adjustment of the tuning of the loop antenna is necessary to adjust accurately the phases of the currents due to the vertical effect and the loop antenna effect if perfectly phased patterns are desired, but modified patterns of various types are very readily secured. Some of these modified patterns are shown in figure 3 , the blunted minima being due to a phase angle between the vertical effect and loop antenna effect. If any one of these field patterns be rapidly reversed so that two equivalent field patterns are produced alternately (figure 3 (d)), and if a zero-center course-indicating meter be made to deflect to the right in proportion to one field pattern, and to the left in proportion to the other field pattern, bilateral indication of direction results. It is obvious that the two positions giving zero or "on course" indications on the meter are at right angles to the plane of the loop antenna and $180^{\circ}$ apart, since each field pattern is symmetrical about the line representing the loop antenna. 
The field pattern of the loop antenna is both modified and reversed by grounding the ends of the loop antenna through two rectifier tubes to which an alternating voltage is applied so that they pass current alternately. When one rectifier passes current its resistance is low, and the loop antenna is effectively grounded at that end. The loop antenna is tuned, and the antenna effect made partially aperiodic by the resistance of the rectifiers.

The course-indicating meter is connected in the cathode return leads of the rectifier tubes so that the currents of the rectifiers pass through it in opposite directions, and give a zero reading. The audio output of the direction finder is applied to the rectifier tubes so that the currents produced by this output voltage are proportional to the corresponding field patterns, and pass through the course indicating: meter in opposite directions. The course indicator then indicates the differences in the voltages developed by the two field patterns, and gives bilateral indication of "course."

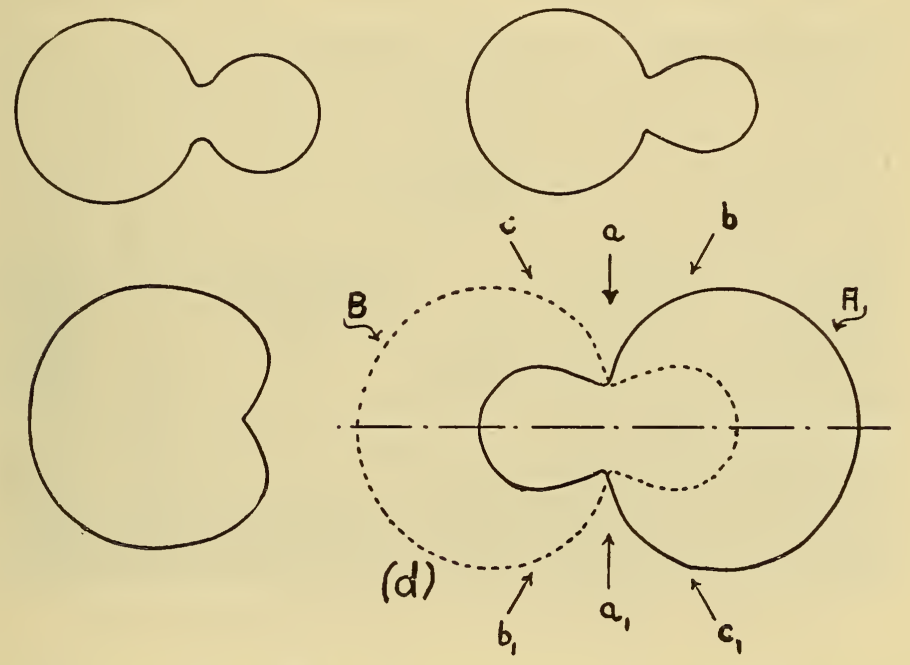

FIGURE 3.-Loop antenna field patterns modified by a vertical component.

\section{THE CIRCUIT ARRANGEMENT}

The circuit arrangement is given in figure 4. Condenser $C_{1}$ tunes loop antenna $L_{1}$ and any voltage developed in the loop antenna by an incoming radio wave is applied to the first radio-frequency amplifier between the center point of the loop antenna and ground. The loop antenna is connected at each end to rectifier tubes $T_{1}$ and $T_{2}$ through equal condensers $C_{2}$ and $C_{3}$. An alternating voltage of a frequency readily passed by the audio amplifier of the radio receiver is applied in opposite phase through radio-frequency inductors $L_{2}$ and $L_{3}$ to the plates of the rectifier tubes. The cathodes of the rectifier tubes are connected one to each end of resistor $R_{1}$, the adjustable center tap of which passes through the audio output transformer of the radio receiver to ground. The zero-center course-indicating meter $M$ is connected across this resistor. Condenser $C_{5}$ is a highcapacity low-voltage electrolytic condenser which damps course 
indicator $M$. An audio oscillator consisting of oscillation transformer $O$ and vacuum tube $T_{4}$ with tuning condenser $C_{4}$ supplies voltage to rectifiers $T_{1}$ and $T_{2}$ across voltage divider $R_{2} R_{3} . \quad R_{2}$ and $R_{3}$ are equal resistors accurately matched to about one half of 1 percent to insure equal voltages of opposite phase for rectifiers $T_{1}$ and $T_{2}$. $R_{4}$ may be added to the circuit to reduce the effect of any changes in the resistance of the rectifier tubes. Greater sensitivity will result if it is omitted. $R_{5}$ and $R_{6}$ are biasing resistors, and $R_{7}$ provides a grid return for first radio-frequency amplifier $T_{3}$. Output transformer $\mathrm{O}_{2}$ applies the output of the radio receiver to rectifier tubes $T_{1}$ and $T_{2}$ and allows them to operate as the output switching device in addition to their function of alternately grounding the ends of the loop antenna.

From figure 4, when a positive voltage is applied to the plate of $T_{1}$ by the audio oscillator, a negative voltage is applied to the plate of $T_{2}$. Then $T_{1}$ draws current and has low resistance to ground, and $T_{2}$ is cut off with high resistance to ground. When the phase of the

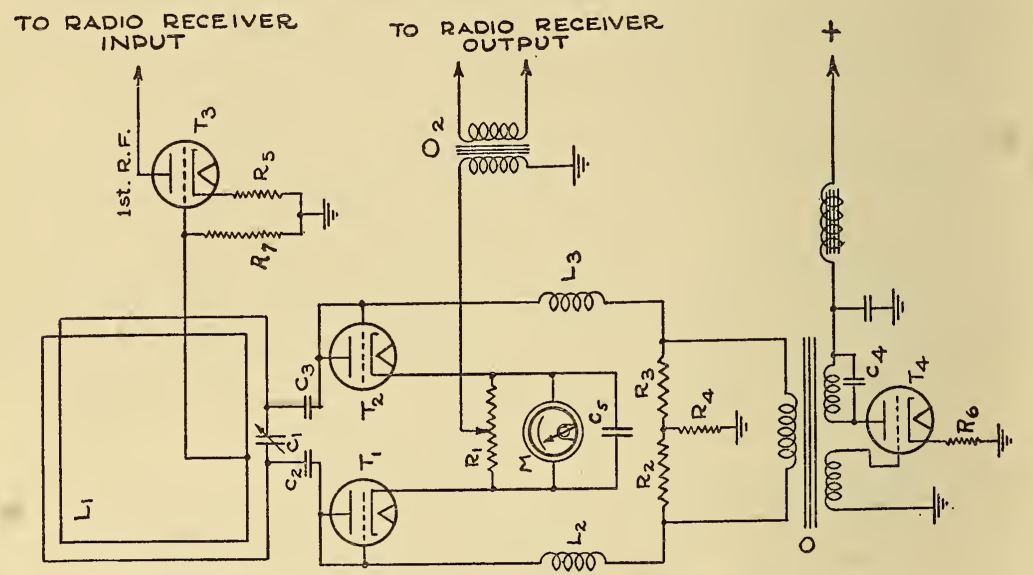

FIGURE 4.-The single loop antenna radio direction finder.

applied voltage reverses, $T_{1}$ is cut off and $T_{2}$ draws current, and the grounding point of the loop antenna is reversed about its center point. Thus the field pattern of the loop antenna is rapidly reversed, and dissymmetry is obtained by placing the ends of the loop antenna alternately at ground.

The currents of $T_{1}$ and $T_{2}$ produced by the voltage of the audio oscillator pass through course indicator $M$ in opposite directions. Since the voltage of the audio oscillator is applied equally to these rectifiers, the currents are equal and the course indicator reads zero. Remembering that when $T_{1}$ passes current one loop antenna field pattern is produced and current passes through the course indicator in one direction, and when $T_{2}$ passes current another field pattern is produced and current passes through the course indicator in the other direction, consider the effect of an incoming radio wave. When the loop antenna is grounded at one end, the signal at the output of the radio receiver is proportional to the field pattern for that condition and the current through the course indicator is proportional to the 


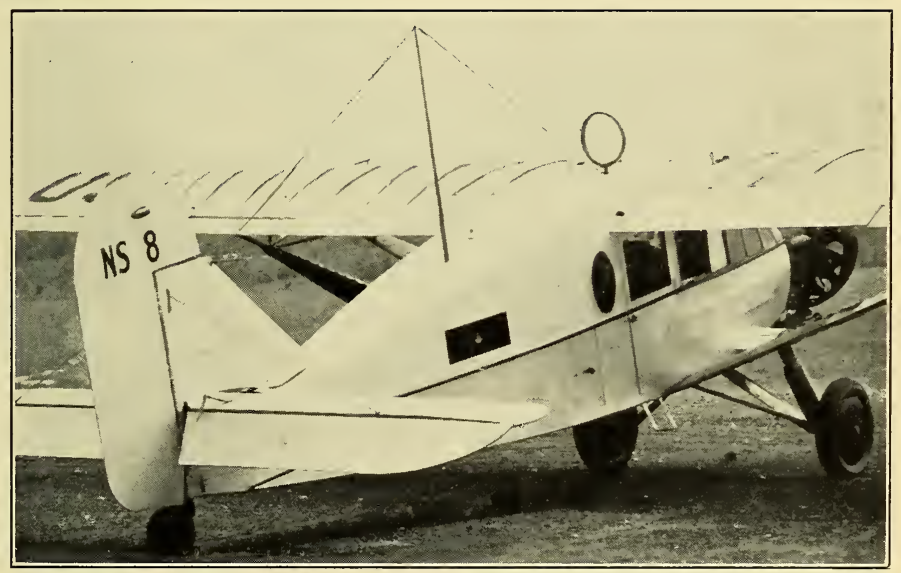

Figure 5.-Loop antenna installed on airplane. 
B.S. Journal of Research, RP621

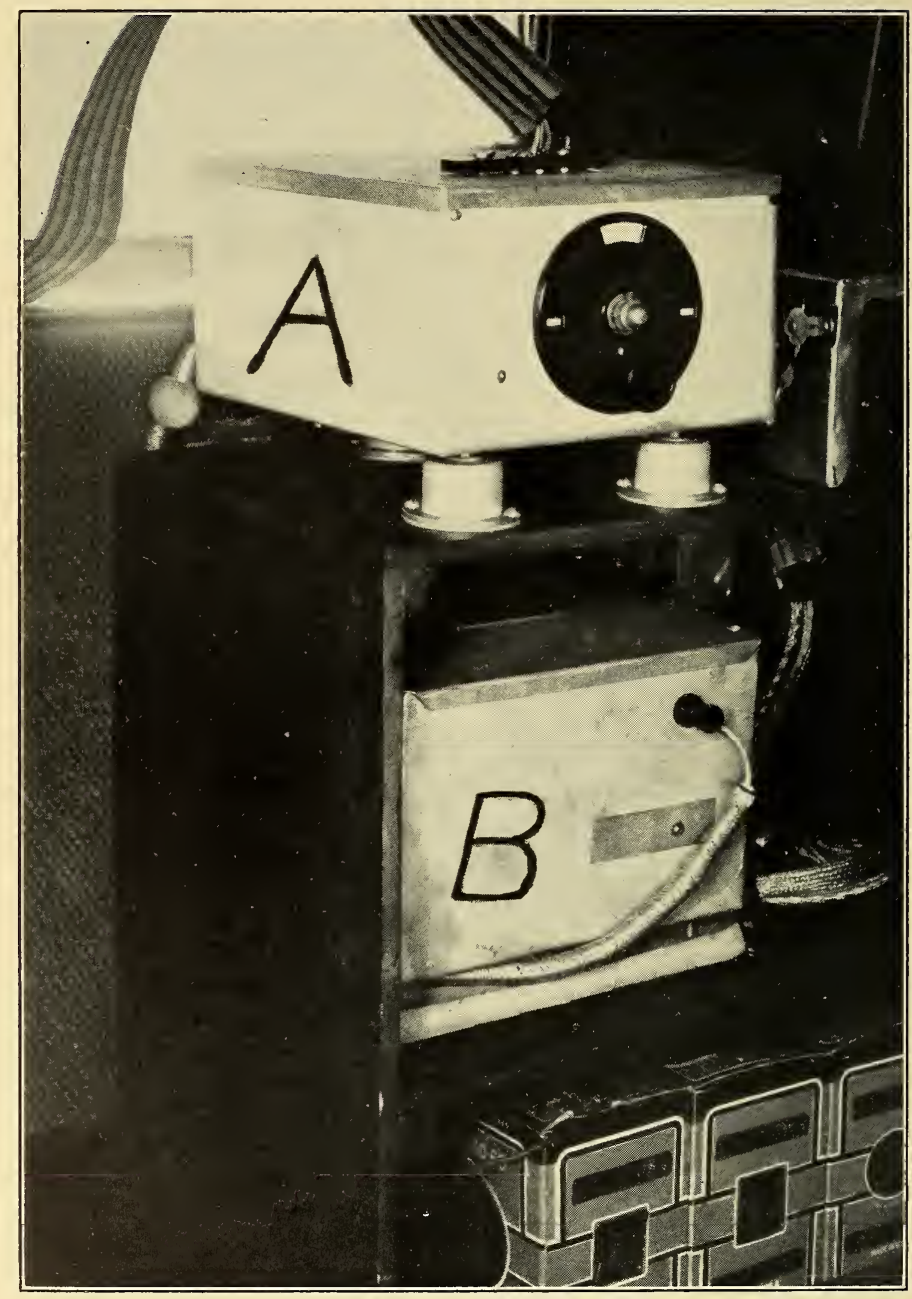

FIGURE 6.-Single-loop antenna direction finder unit $A$ and radio receiver $B$ installed for test in airplane. 
voltage of the audio oscillator and the output voltage. When the loop antenna is grounded at the other end similar conditions hold, but there is a reversal of current in the course indicator. Since the currents through the course indicator due to the audio oscillator are equal and opposite, currents deflecting the course indicator right or left are directly proportional to the difference between the output voltages due to each field pattern.

There are two positions of the loop antenna with respect to an incoming radio wave which gives "on-course" indications. These two positions are at right angles to the plane of the loop antenna and $180^{\circ}$ apart. It can be seen from figure 3 (d) that the action of the course indicator for one course is the reverse of its action for the other course, giving automatic directive sense. Let $A$ and $B$ be the field patterns developed by the loop antenna, the course indicator deflecting to the right when $A$ is produced, and to the left when $B$ is produced. When a radio wave is received from direction $a$ the field patterns are equal and "on course" reading results. If the radio wave comes from direction $b, A$ is much larger than $B$, and the course indicator reads to the right of "on course." The loop antenna must then be rotated to the right for an "on course" indication. Similarly, a signal from direction $a_{1}$ gives an "on course" indication, but a signal from $b_{1}$ produces a reading to the left of "on course", which is the reverse of the indication for a signal from direction $b$. By following the rule that when heading toward the source of the signal the indicator deflects in the direction the loop antenna is "off course", directive "sense" is assured.

\section{CONSTRUCTION}

In the construction of the direction finder, care must be taken that the loop antenna is symmetrical about its center point with respect to ground. There should be no displacement current or secondary loop antenna effect. This is avoided by using a narrow type of winding in the loop antenna. The plate circuits of the two rectifiers must also be balanced for equal inductance and capacity. A slight distortion of "course" results when these circuits are not balanced. The loop antenna may be mounted at a distance from the radio receiver and the direction-finder unit, but the leads connecting these units should be fixed in position by a web belt or some similar means, and the capacity to ground should be low. Resistors $R_{2}$ and $R_{3}$ must be matched to about one half of 1 percent. Type 237 tubes are used as rectifiers, the grids and plates being connected together. Variation in the resistance of these tubes has little effect on the field pattern produced. In the output circuit, since it depends on the balancing of currents, the rectifiers should be fairly well matched. $R_{1}$ serves to complete the matching of the rectifiers. It is adjusted for zero reading of the course indicator when no signal is being received.

\section{TESTS}

The direction finder has been installed and tested on an airplane at College Park. A photograph showing the installation of the loop antenna on the airplane is given in figure 5, and the direction-finder unit with the radio receiver is shown in figure 6 . The direction-finder unit $A$ with the belt connection to the loop antenna is at the upper 
left of the installation, and the control unit for the radio receiver is mounted beside it. The radio receiver $B$ is directly below the direction-finder unit. Figure 7 shows the course indicator. Test flights were made in the vicinity of Washington and Baltimore, using the broadcasting stations of those two cities. In these tests the direction finder was used as a homing device. Its operation was entirely satisfactory, indications right and left of "course" being very steady and definite. On passing over the stations toward which the flights were made, positive localizing action was given by the reversal of the action of the course indicator. Although the locations of the various stations

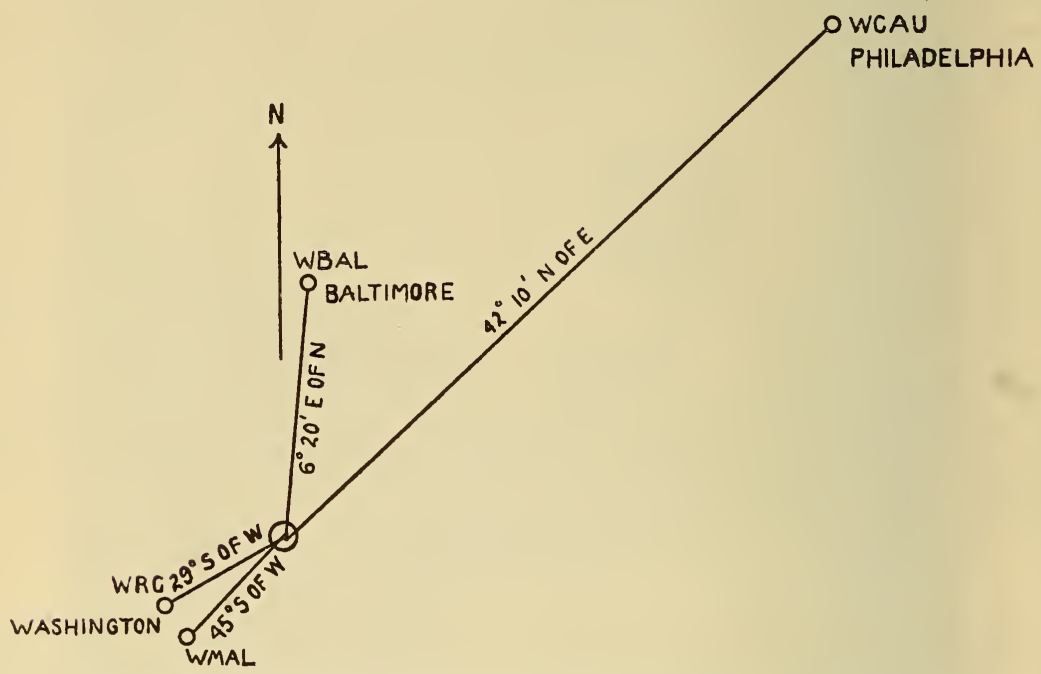

Figure 8.-Typical direction-finder bearings taken at College Park.

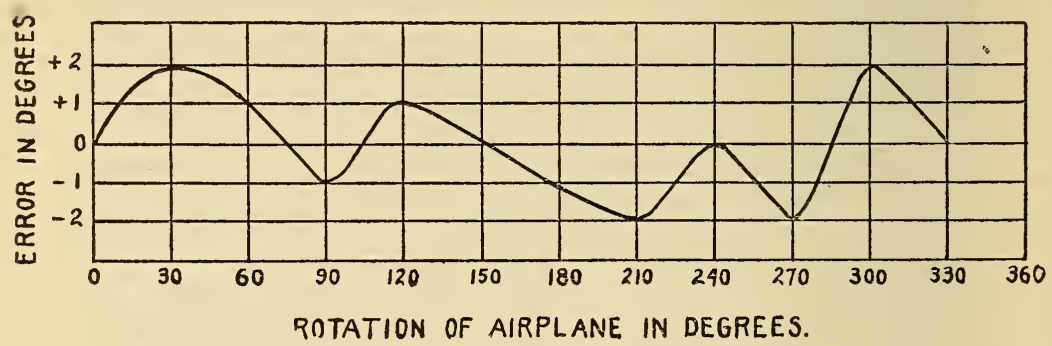

FIGURE 9.-Calibration graph for errors introduced by airplane structure.

were unknown to the pilot in each case, he immediately located the station to within a half mile radius from an altitude of about 3,000 feet.

Figure 8 shows triangulation data taken on the ground at College Park, Md. A magnetic compass was used as a basis for the measurements. Figure 9 gives the error in indication for the loop antenna installation on the airplane.

The accuracy of the direction finder is of the order of $1^{\circ}$. The sensitivity of the indications varies with volume, and it can be very easily ncreased or decreased by changing the circuit constants. Fullscale deflection of the course indicator for $10^{\circ}$ variation from "course" 


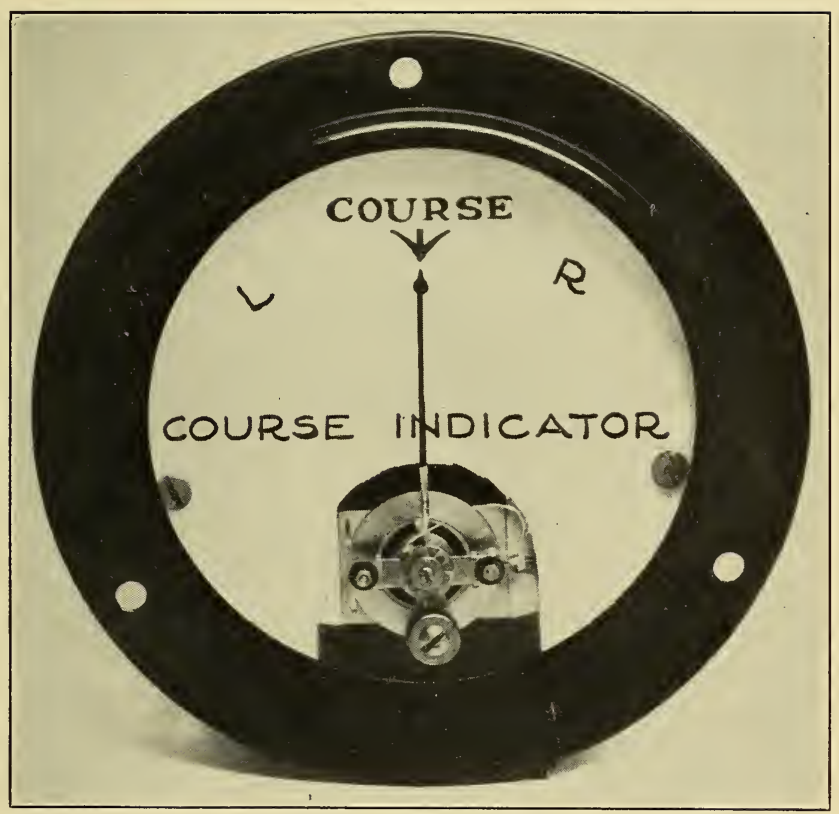

Figure 7.-Course indicator. 
is readily secured. No distortion of "course" could be noticed at any volume level, including complete overloading of the radio receiver. An interesting feature of the direction finder is its action under conditions of bad static or ignition interference. When "on-course" signals are received there is little effect, and when "off-course" signals are received any change in indication tends toward an "on-course" indication. No violent kicking of the course indicator occurs.

Signals are intelligible while the direction finder is operating. When "on course", only a constant tone of low intensity being present in the radio receiver. When "off-course" indications are received, this tone increases in intensity, tending to destroy the characteristics of the incoming signal. Aural check on the course indication is thus given.

The direction finder operates equally well on modulated or unmodulated waves. When the radio wave is intermittent, the direction finder operates only when the radio wave is received. In this case indications right and left of "course" are given by a kicking of the course indicator right and left from zero. The "on-course" indication is constant.

There is practically no tuning error if the loop antenna is resonated at, or above, the frequency of the station on which the bearing is taken. If the loop antenna is resonated below this frequency, the ficld patterns may be badly distorted by the reversal of phase of the loop-antenna current, thus producing course errors. The loop antenna, then, may either be tuned or untuned, providing that its resonant frequency is never lower than that of the station on which the bearing is being taken.

\section{ACKNOWLEDGMENT}

Acknowledgment is made to $\mathrm{H}$. Diamond for basic contributions in some of the earlier models, and for many helpful suggestions in connection with the final development, and to L. L. Hughes for skill in construction of the various models. G. H. Lester gave valuable assistance in the construction and testing of the final model.

Washington, July 20, 1933. 